\title{
On Eyal Peretz's Becoming Visionary
}

The following comments, touched up here and there and slightly amplified, were my response to an invitation to supply a reader's report to Stanford University Press in view of their interest in publishing this remarkable book. I am accepting the sense expressed by both Eyal Peretz and by the book's editor, Norris Pope, that the appearance of my comments together with the book may encourage productive conversation with it.

Peretz's book on a small set of films of Brian De Palma is openly and triply ambitious. First, it seeks to show that this well-known filmmaker, about whom in my hearing it is rather taken for granted that he is no more than a gifted sensationalist and imitator of, especially, Hitchcock, is instead an artist and thinker in film who ranks in originality and depth with the greatest directors in the history of sound film. Second, it accepts the challenge that to show this requires a new perception and altered conceptualization of what one is to understand by the history of film. Third, it asserts that this new understanding requires an invocation not of some more or less vague invocation of a "theory" of film, but precisely the tracing of an intersection of film with philosophy, which in turn requires, or has required, a reconception, even a kind of overcoming, of philosophy as it stands. (One might include as a fourth ambition the demonstration that these three tasks are, or should be, part of the same intellectual effort.) The ambition is bound to seem preposterously vast. This in itself should not, however, seem very surprising where philosophy comes insistently into an argumentative picture: As I have had occasion variously to insist, philosophy is inherently arrogant, arrogating to itself the power of speaking universally, speaking for all (all who will hear), without claiming (indeed disclaiming access to) knowledge that the rest of the world does not possess (those who merely do not know that they possess it). (The arrogance is just as relentless in the tones of J. L. Austin and of Wittgenstein in 
their demand for the ordinary as it is in the culture of European sages who behave as if the ordinary is itself a source of horror. I do not say that the latter are perfectly wrong in this. In a sense Austin and Wittgenstein equally insist upon it.) What is really surprising is the extent to which Peretz succeeds in realizing his extravagant ambitions.

Something I must regard as a limitation on this success is internal to the condition that makes Peretz's success possible for him. He does to my mind show and articulate an irreducible sense in which film and philosophy call upon each other, a task close to my heart, one without which, I am convinced, philosophy as well as film is deprived of an essential register of contemporary effect and understanding. But I take it as a fact of contemporary intellectual life that philosophy continues to exist in two states, to some perhaps irreducible extent unintelligible to each other. The mode of philosophy Peretz invokes and unquestioningly accepts and puts into play, represents one half of the split contemporary philosophical mind. It is a brilliant half, explicitly in Peretz's text including the names of Blanchot, Deleuze, Derrida, Lacan, Lyotard, Nancy, etc.-so brilliant indeed that the idea of a mere "half" of philosophy invoked by Peretz will seem to some drastically understated. For two particular reasons. First, the opposite half of the philosophical mind, poorly named analytical philosophy and poorly identified with England and the United States, leading figures in one of its branches being Wittgenstein and Austin, in the other branch Carnap and Quine and Donald Davidson and Hilary Putnam, has developed comparatively little that is apparently useful to the understanding of film, or indeed to the arts more generally. Second, Peretz's intense and precise readings of individual films reflects back upon the philosophy with which Peretz demonstrates its encounter and thus brings new and surprising illumination and comprehension to that philosophical strain.

This second reason emphasizes the deeply pedagogical intention and achievement of Peretz's text. Its analysis of individual films are among the deepest known to me, tireless and surprising to the end, unswervingly following the experience or conviction that film, in its high instances (more numerous than a hasty glance announces), deserves the attention due to, rewarded by, a great art. (Writers-apart from those who have wonderfully devoted an occasional essay to a film-whom I have found repeatedly to reach and articulate this depth in the experience of individual films, on the other side of the English Channel, and without heavy explicit attention to 
philosophy, are Victor Perkins and Andrew Klevan, and on this side of the Atlantic, William Rothman, who along with this critical intensity remains in touch with his analytical philosophical formation.) And Peretz's management of the philosophical tradition in which he places his confidence is not the result of fashion (the mere fashion of the thing, while for a couple of decades world-consuming, seems to be past), but of years of hard and intelligent and subtle and fruitful immersion in working with it. $\mathrm{He}$ is by training and beyond the evidence of his writing a thoroughly trustworthy teacher of this material, moreover one to whose value students readily attest. (To one not accustomed to the idea that films can be shown to reveal the dimensions of a great art on the wings of recent decades of French philosophizing, I recommend a look at Peretz's superb study of Moby Dick, a revision of his doctoral dissertation, where the surprising intersection of that thinking with a text whose greatness few would deny is a feat whose success is perhaps initially easier to see and accept.) So good are Peretz's separate readings, indeed, that they form a serious attestation of the fact of film, an understanding of the cinematic medium, that does justice to the reach of its experience, hence to be a ground upon which the two traditions of philosophy have a reasonable chance for intelligible encounter and precious exchange.

I realize that such an encounter, while of key importance to me, is not high on everyone's hopes for a future of academic humanistic work. So I shall sketch my own stake in my hopes for the success of Peretz's film book. Here I must allow myself a certain latitude of explicit immodesty, namely in assuming that the work I have published on film counts as some contribution to a field that might be called Film and Philosophy. (This is a title I have been hearing for the past year or two. Early this year, after a lecture I gave on film at the École Normale in Paris, where I have lectured on various occasions, but not for several years now, I was surprised by the standing room audience, and afterward at dinner I asked the professor sitting across from me where this evidently increased interest in film has come from. He replied that he and others had initiated a program in film at the École Normale prompted by student demand for this addition to the curriculum. I said I could not imagine that this meant the École plans to offer courses in filmmaking. "Of course not. The program is called Film and Philosophy." I was startled and I asked what that title includes. "Well, it is Deleuze and you." It was not the first time in recent months that I had 
heard this pairing in the context of comments noting how few philosophers who publish with reasonable regularity within the realm of philosophy also publish multiply about film. (I cannot accept this state of affairs as a mere curiosity. I find it, supposing the claim is roughly true, depressing and anything but easy to comprehend, given the unprecedented and worldwide effects the invention and the achievements of cinema have had on the means and ends of the traditional great arts.) Peretz registers a comparable perception in the course of a long footnote (Chapter 2, Note 37) in which he explains why he is developing Deleuze's idea of a frame rather than the related idea of it in my work, especially in The World Viewed.

A cautionary word here. My interlocutor from the École Normale may in his identification of the field to be known as Film and Philosophy have simply been showing courtesy to a visiting lecturer. Or he may conceivably have meant something quite different, something that in the States I see announced more or less as Philosophy and Film, represented for example in a substantial proposed anthology which puts together selections from the history of philosophy and from recent analytical philosophy arranged by topic (philosophy of mind; ethics; free will, etc.) and suggests in each case segments from films that illustrate the topic, promising thereby increased student interest in learning of and discussing these established issues. No problem here, and talented teachers may thereby achieve heightened classroom results; one could even say that philosophy has been pedagogically slow in making use of what we used to call audiovisual materials. A way to put the difference in what I might like to see become the field of Film and Philosophy, anyway in how I have conceived my writing on film to be motivated philosophically, is that it takes the fact of film itself to become a challenge for philosophy. (It may be the inkling that film possess this power that from its beginning has caused philosophers, with rare exceptions, to avoid the subject.) Cinema's bursting onto the artistic and intellectual scene full-blown, in the second decade of the twentieth century, scarcely twenty years after its technological realization, joined in questioning the concept of art, of modernism, of narrative, of character, of perception, of intention, of entertainment, of identification, indeed of every concept touching the region of the aesthetic, to go no farther. I say immediately that if there is such a field in the offing as Film and Philosophy, so conceived, Peretz's book must form a notable piece of its curriculum. 
Peretz is quite explicit about a new intersection called upon by the art of film and the practice of philosophy. In the last pages of the longest essay in his book, that on De Palma's Blow Out, Peretz describes and instances a gesture he recurs to throughout his text: "Once again, by resorting to a fundamental image in the history of philosophy, we can illuminate what is at stake in this final projection in the dark room" (Chapter 3). I pair this with a passage from a lecture I gave ("Something Out of the Ordinary") to the American Philosophical Association in 1996, collected in my Philosophy the Day After Tomorrow: "I am proposing ... , following what I construe Kant's examples of the transgressions of reason, in their intersection with Shakespearean drama, to suggest (perhaps it is Hegel's suggestion) [,] that the arts, beginning with tragedy (or, in Hegel's aesthetics, ending with tragedy), may variously be seen, or claimed, as chapters of the history, or development, of philosophy, hence perhaps of certain of its present manifestations" (p. 14). But this self-evidently close conjunction of perceptions between Peretz and me at the same time underscores differences I have begun to suggest, deriving from our each recognizing responsibilities to sometimes opposed states of present philosophy. (This is something of an exaggeration, perhaps only of interest to me, namely that in my case the two strains are meant to be at work, if still variously at odds, within the same prose.)

My hopefulness prompted by this conjunction of closeness and distance in the philosophical invocations in Peretz's and in my addresses to cinema lies practically, in my reading of Peretz's new book, in my experience that, so often with each new insight Peretz arrives at, I could sense the prompting to another route of arrival from within the texture of concepts deployed in my World Viewed, if not suggesting a coincidence of result then suggesting a difference promising to my mind fruitful lines of variance. Unnecessary incomprehension is something I am always on the lookout for. In my first decade and a half of studying and teaching philosophy, Wittgenstein and Heidegger were regarded as blanks to each other. It was an important turn in my intellectual life when I began to grasp the intimacy of their differences. (In Chapter 2, Note 37, referred to here previously, Peretz locates my work in relation to Deleuze as "more or less of that tradition." The generality of that description captures something important here, but as I suggested a moment ago, I feel it of decisive importance to the way my concerns have unfolded that I was trained 
exclusively in analytical departments of philosophy and that the "other" tradition was one, and remains one, to which my attractions were always deep but always threatened to isolate me and were always in need of justification; eventually this meant that my relation to the analytical tradition stood equally in need of justification.)

If there is an active source of this series of intimate differences between Peretz's and my lines of investigation, more concrete than differing orientations of philosophical practice, it lies in the relation between what Peretz identifies as the excess of experience as expressed in the concept of haunting, and what I characterize as skepticism, which for me is what produces the impulsion toward, a longing for, a beyond, say an excess. This reading of skepticism is something I find dramatized in Wittgenstein's Philosophical Investigations, which distinguishes my reading of Wittgenstein from the rather favored reading which takes him to deny the meaningfulness, or else to provide a refutation, of skepticism. Wittgenstein, according to my reading, should accordingly be included in, or related to, Peretz's observation that "overcoming metaphysical dualisms characterizes every major modern philosophy since Hegel" (p. 129), dualisms namely that are immediate or distant inheritors of Plato's dualism between the sensuous immanent and the intellectual transcendent-where "overcoming" is not a matter of refuting or denying but of what some have called dialectic, others deconstruction, what Austin calls dismantling, what Wittgenstein shows to require alertness to differences among the grammars of concepts. (Wittgenstein, like Emerson, and not unlike Heidegger, seeks to demonstrate how the radical instability of our concepts demands of our lives an unending philosophical vigilance.) Another yet more concrete instance of similarity/difference may be found in Peretz's contrasting Deleuze's and my accounts of what is "beyond" or in excess of the frame by saying that for Deleuze there are two senses of an excess beyond the frame, a continuous and a discontinuous sense, the former an opening into, let's say, the rest of what a given focus leaves outside the frame, adjacent to it, the latter an opening onto an absolute outside, the outside as such. What in The World Viewed I conceive as the absolute outside is the world as such, and what "implies" it on my account is not the frame, but what the frame covers, namely the screen, screening us from the world. The screen is one of the few fundamental facts of the necessary conditions, or automatisms, of film, in contrast with the automatism of the frame (among such facts that 
are articulated in his or in my texts), that Peretz, as I remember, does very little with. But his concentrating on the frame produces unique and indispensable returns. For example, Peretz's consequent discussion of the senses, of their splitting and supplementation through film, is breakthrough material, true advances in understanding.

The division in the contemporary philosophical mind is of its nature not something that most philosophers, or theorists generally, are likely to be distressed by, finding work enough to do within, and perhaps in struggle with, the separate dispensations they have inherited. Since it is not likely, nor desirable, and perhaps not really conceivable, that one side will overcome the other, the task of thinking will for the foreseeable future continue to exist within or between each. I add that my sense of fruitfulness in reading Peretz is not something I have so far managed to derive from reading (to the extent I have been motivated to read) Deleuze's own two books on film. (In a recent conference on what might as well been called Film (or the photographic) and Philosophy, organized by Michael Fried, I was moved in response to one paper to declare that I would now read through Deleuze on cinema, which various attending voices found to be a rash promise.) Certainly a decisive cause of this difference is the fact of the countless conversations about film and literature that Eyal Peretz and I have had in the course of our acquaintance and friendship over the past decade. But an accompanying cause is a signal difference between the practices of Peretz and (to the extent that I am aware of them) of Deleuze, in their considerations of film, namely that Peretz's theoretical claims are motivated by a persistent uncovering of the workings of a few individual films, as if following a wager that neither a progress of theory nor a disclosure of the depths of a significant work of film will be the first to exhaust itself. There were times when I felt some editing would be able to remove a certain repetitiveness in Peretz's accounts, but each time I changed my mind. The repetitions express Peretz's sense of pedagogical necessity, and I can see that they make possible the certain cascades of surprises that Peretz likes to unleash. In the light of his achievement I find plenty of work cut out for me. Not for me alone I trust. 
